\title{
ON THE CAYLEY-VERONESE CLASS OF CONFIGURATIONS*
}

\author{
BY \\ WALTER B. CARVER
}

CaYley, in his paper Sur quelques théorèmes de la géomètrie de position, $\dagger$ first calls attention to the figures obtained by taking the section, by a plane or 3-dimensional space, of the complete $n$-point (viz., $n$ points, and the $\left(\begin{array}{l}n \\ 2\end{array}\right)$ lines, $\left(\begin{array}{l}n \\ 3\end{array}\right)$ planes, etc., determined by them) in a flat space of $\nu$ dimensions.

Later, VERONESE discusses more fully $\ddagger$ the nature of this class of configurations, treating in general the configurations thus obtained in $r$ dimensions. Both Cayley and Veronese state that these same configurations can also be obtained as projections of higher-dimensional figures.

Among the posthumous papers of Caporali is a paper: Sulla teoria delle configurazioni $\S(1879)$, giving, without proof, a number of theorems concerning a certain class of plane configurations. Although there is no mention of space of higher dimensions in CAPORALI's paper, his configurations may be regarded as projections upon a plane, or sections by a plane, of simple higher-dimensional figures; and they belong, in fact, to the CAYLEY-Veronese class indicated above. CaPORALI's theorems become almost self-evident when viewed from this standpoint.

In a paper Ueber Polyedrale Configurationen, $\|$ DE VRIEs discusses a special sub-class of the Cayley-Veronese configurations, viz., the plane sections of complete $n$-points in ordinary space.

It is my purpose in this paper to state a number of general theorems for the Cayley-Veronese configurations in space of $r$-dimensions (extensions, for the most part, of Caporali's theorems for the plane case); to give a construction for the quadric polarity in the plane and in 3-dimensional space, based upon certain of these configurations; and finally to call attention to certain peculiar sets of conics in the plane, connected with plane configurations of this class. \

\footnotetext{
* Received for publication, August 23, 1904. Presented to the Society October 28, 1905.

†Crelle's Journal, vol. 31 (1846); Collected Papers, vol. 1, p. 317.

$\ddagger$ Behandlung der projectivischen Verliältnisse der Räume von verschiedenen Dimensionen durch das Princip des Projicirens und Schneidens, Mathematische Annalen, vol. 19 (1882).

\$Memorie di Geometria, p. 262.

Mathematische Annalen, vol. 34 (1889).

T There may be mentioned a few of the very many instances of the incidental occurrence of these configurations.

The well-known figure of two perspective triangles with their center and axis of perspection
} 
In this paper, $S_{\nu}$ denotes, as usual, a linear or flat space of $\nu$ dimensions (in points). The words " copoint," " coplane," etc., are used to specify the dual of the point, plane, etc., in the space $S_{v}$; thus, in $S_{\nu}$, a copoint is an $S_{v-1}$, and a complete $n$-copoint is the dual of the complete $n$-point. The number of combinations of $n$-things taken $\nu$ at a time will be denoted by $\left(\begin{array}{l}n \\ \nu\end{array}\right)$, with the usual convention that $\left(\begin{array}{l}n \\ n\end{array}\right)=\left(\begin{array}{l}n \\ n\end{array}\right)=1$.

\section{§1. General Theorems.}

Let $\Gamma_{n, r}^{\nu}$ dencte the configuration obtained in an $S_{r}$ by cutting a complete $n$-point in $S_{\nu}$ by the $S_{r}$. The incidence relations of the configuration* may be shown by means of the square matrix of the $r$ th order

$$
\left(\alpha_{p q}\right) \quad(p, q=0,1, \cdots, r-1)
$$

in which $\alpha_{p p}$ denotes the number of $S_{p}$ 's in the configuration, and $\alpha_{p q}(p \neq q)$ denotes the number of $S_{p}^{\prime}$ 's incident with each $S_{q} \cdot \dagger$ For the $\Gamma_{n, r}^{\nu}$ we have

$$
\begin{aligned}
& a_{p p}=\left(\begin{array}{c}
n+n \\
p+\nu-r+1
\end{array}\right), \\
& a_{p q}=\left(\begin{array}{c}
q+\nu-r+1 \\
p+\nu-r-1
\end{array}\right) \\
& \alpha_{p q}=\left(\begin{array}{c}
n-q-\nu+r-1 \\
p-q
\end{array}\right)
\end{aligned}
$$$$
(p>q)
$$

If each of the $n$-points in $S_{\nu}$ is denoted by a single letter, any $S_{p}$ of the $\Gamma_{n, r}^{\nu}$ is denoted by a combination of $p+\nu-r+1$ of these $n$ letters; and any particular $S_{p}$ and $S_{q}(p>q)$ of the configuration are incident if the $q+\nu-r+1$

belongs to this class. It was probably first mentioued by Desargurs in 1636 (cf. ENESTröm, Bibliotheca Mathematica, 1885), and is discuesed in the aforementioned paper of CAYLEY, and also in KaNTOR's paper on $(3,3)_{10}$ Configurations, Sitzungsberichte der K. A kad., W i en, vol. 84 (1881). Veronese shows, in his memoirs, Nuovi teoremi sull' hexagrammum mysticum, Atti della R. Accad. dei Lincei, vol. 1 (1877), and Interprétations géometriques de la theorie des substitutions de n-letters, etc., Annali di Mathematica, vol. 11 (1881), that the sixty lines of the Pascal hexagram form six of these Desargues figures.

The confignation of two perspective tetrahedra with their center and plane of perspection occurs in KLEIN's memoir, Zur Theorie der Liniencomplexe des ersten und zweiten Grades, Math ematisohe Annalen, vol. 2 (1870); and also in RICHMond's paper On the figure of six points in space of four dimensions, Quarterly Jonrnal of Pure aud Applied Matbematics, vol. 31 (1900).

WHITEHEAD treats of the figure of two perspective reference figures in space of any number of dimensions (Universal Algebra, vol. 1, p. 139).

CaYLEY, loc. cit., shows that a peculiar special case of the plane figure of fifteen lines and twenty points, obtained as a section of the figure of two perspective tetrahedra, occurs in the Pascal hexagram.

* Cf. Veronese, Mathematische Annalen, vol. 19 (1882), p. 171; or Grundzüge der Geometric, p. 615.

† This is the notation suggested by Professor Moore, Tactical Memoranda, American Journal of Mathematics, vol. 18 (1896), p. 265. 
letters belonging to the latter are contained among the $p+\nu-r+1$ letters belonging to the former.

Suppose, on the other hand, that one starts with the complete n-copoint in $S_{\nu}$, and projects it upon an $S_{r}$, taking a co- $S_{r}$, or $S_{\nu-r+1}$, as the projectioncenter. By such a projection,* each point, line, plane, $\ldots$ or $S_{r-1}$ of the original figure is sent into a new point, line, plane, $\cdots$ or $S_{r-1}$ in the $S_{r}$. The $S_{r}^{\prime}$ 's, $S_{r+1}$ 's, $\cdots$ and $S_{v-1}$ 's of the original figure are lost. The configuration thus obtained in the $S_{r}$ is evidently the dual of the $\Gamma_{n, r}^{\nu}$, and may be represented by the symbol, $C_{n, r}^{v}$.

The iucidence relations of the $C_{n, r}^{v}$ may be shown by the matrix

where

$$
\left(a_{p q}\right) \quad(p, q=0,1, \cdots, r-1)
$$

$$
\begin{array}{ll}
\dot{a}_{p p}=\left(\begin{array}{l}
n \\
\nu-p
\end{array}\right), & \\
a_{p q}=\left(\begin{array}{c}
n-\nu+q \\
q-p
\end{array}\right), & (q>p), \\
a_{p q}=\left(\begin{array}{c}
\nu-q \\
\nu-p
\end{array}\right), & (p>q) .
\end{array}
$$

Any element, $S_{p}$, of a $C_{n, r}^{\nu}$ is named by a combination of $\nu-p$ letters out of $n$. If we re-name all the elements, assigning as a new name to each $S_{p}$ the $n-\nu+p$ letters not contained in its old name, we see at once that the $C_{n, r}^{\nu}$ under this new lettering becomes a $\Gamma_{n, r}^{n-\nu+r-1}$. A $\Gamma_{n, r}^{\nu}$, with a similar change of lettering, becomes a $C_{n, r}^{n-\nu+r-1}$. (It will be convenient hereafter to write $\mu$ for $n-\nu+r-1$.) Since a $\Gamma_{n, r}^{\nu}$ and a $C_{n, r}^{\nu}$ are dual figures, it follows that a $C_{n, r}^{\nu}$ and a $C_{n, r}^{\mu}$ are dual, as are also a $\Gamma_{n, r}^{\nu}$ and a $\Gamma_{n, r}^{\mu}$. Also a $\Gamma_{n, r}^{\nu}$ or a $C_{n, r}^{\nu}$ is self-dual if $n-\nu+r-1=\nu$, i. e., if $2 \nu=n+r-1$. $\dagger$

While the symbols $C_{n, r}^{\nu}$ and $\Gamma_{n, r}^{\mu}$ represent the same configuration, it is convenient to have both. One may use $\Gamma_{n, r}^{\mu}$, when considering the figure as a section of a $\mu$-dimensional figure, and $C_{n, r}^{\nu}$ when regarding it as a projection of a $\nu$-dimensional figure. $\ddagger$

Theorem I. If in a $C_{n, r}^{\nu}$ we separate all those elements whose names contain a certain letter from those whose names do not contain it, the latter form $a C_{n-1, r}^{\nu}$ and the former $a C_{n-1, r}^{\nu}$. Each $S_{p}$ of the $C_{n-1, r}^{\nu}(p=1,2,3, \ldots, r-1)$ is incident with an $S_{p-1}$ of the $C_{n-1, r}^{\nu-1}$.

* This is what Veron ase calls an eindeutige projection. C. Grundzüge der Geometrie, p. 614.

†C. Vhronkse, loo. cit., p. 164 ; also Grundzüge der Geometrie, p. 623.

$\ddagger$ These configurations may, of course, be defined without reference to space of higher dimensions than the $S_{r}$ in which they lie; but it should be understood that the matrix giving their incidence relations is not sufficient so to define them. We must add the condition that they be so arranged that they can be lettered, $p+v-r+1$ letters to each $S_{p}$, in such a way that the $q+v-r+1$ letters of any $S_{q}$ incident with a given $S_{p}(p>q)$ shall be contained among the $p+v-r+1$ letters of the $S_{p}$. 
When a $C_{n-1, r}^{\nu}$ and a $C_{n-1, r}^{\nu-1}$ are so related we may speak of them as being chiastically incident, or, for present purposes, simply as chiastic.*

Theorem II. With respect to two letters, say $a$ and $b$, the $C_{n, r}^{v}$ breaks up into a $C_{n-2, r}^{\nu}$, the names of whose elements contain neither a nor $b$; two $C_{n-2, r}^{\nu-1} \cdot s$, one containing $a$ but not $b$, the other $b$ but not $a$; and $a C_{n-2, r}^{\nu-2}$ containing both $a$ and $b$.

The two $C_{n-2, r}^{\nu-1}$ 's are both chiastic with the $C_{n-2, r}^{\nu}$ and also with the $C_{n-2, r}^{\nu-2}$. Moreover each $S_{p}$ of the $C_{n-2, r}^{\nu}(p=2,3, \ldots, r-1)$ is incident with an $S_{p-2}$ of the $C_{n-2, r}^{\nu-2}$; and we may speak of a $C_{n-2, r}^{\nu}$ and a $C_{n-2, r}^{\nu-2}$ so related as being chiastic.

In general, any two configurations whose symbols have the same subscripts but different superscripts, as $C_{n, r}^{\nu}$ and $C_{n, r}^{\nu-\kappa}$, are chiastic if each $S_{p}$ of the $C_{n, r}^{\nu}$ is incident (in a certain order) with an $S_{p-\kappa}$ of the $C_{n, r}^{\nu-\kappa}$. It is evident that we cannot have chiasm between these two figures unless $\kappa \leqq r-1$. If a $C_{n, r}^{\nu-\kappa}$ is chiastic with a $C_{n, r}^{\nu}$, and the $C_{n, r}^{\nu}$ is chiastic with a $C_{n, r}^{\nu+l}$, then the $C_{n, r}^{\nu-\kappa}$ is chiastic with the $C_{n, r}^{\nu+l}$.

THEOREM III. The breaking up of a $C_{n, r}^{v}$ with respect to $S$ given letters may be symbolically expressed by the formula:

$$
C_{n, r}^{\nu} \equiv C_{n-s, r}^{\nu}+s C_{n-8, r}^{\nu-1}+\left(\begin{array}{l}
8 \\
2
\end{array}\right) C_{n-8, r}^{\nu-2}+\cdots+\left(\begin{array}{l}
8 \\
2
\end{array}\right) C_{n-8, r}^{\nu-8+2}+s C_{n-\delta, r}^{\nu-s+1}+C_{n-8, r}^{\nu-s} .
$$

Any $C_{n-s, r}^{\nu-\kappa}$ of this expression is made up of those elements of the $C_{n, r}^{v}$ whose symbols contain a certain $\kappa$ of the $s$ letters but not the remaining $s-\kappa$. A $C_{n-s, r}^{\nu-\kappa}$ and a $C_{n-s, r}^{\nu-\kappa+l}$ are chiastic if the $\kappa-l$ letters which distinguish the latter are contained among the $\kappa$ letters which distinguish the former.

Theorem IV. There are $\left(\begin{array}{l}n \\ s\end{array}\right)\left(\begin{array}{l}8 \\ \kappa\end{array}\right) C_{n-s, r}^{\nu-\kappa}, s$ contained in every $C_{n, r}^{v}$.

Theonem V. If $s C_{n-s, r}^{\nu-1}$ 's are chiastic with a $C_{n-\delta, r}^{\nu}$, any two of the $C_{n-8, r}^{\nu-1}$ 's determine a $C_{n-s, r}^{\nu-2}$ with which they are both chiastic; three of the $C_{n \rightarrow-, r}^{\nu-1}$ 's determine thus three $C_{n-8, r}^{\nu-2}$ 's which are chiastic with and deterinine a $C_{n-s, r}^{\nu-3} ; \cdots$ and finally, the $s C_{n-s, r}^{\nu-1}$ 's determine $s C_{n-s, r}^{\nu-s+1}$ 's which are chiastic with and determine $a C_{n-s, r}^{\nu-s}$; and the entire figure thus determined is a $C_{n, r}^{\nu}$.

When $\nu=n-s$, this theorem is Veronese's "perspective pyramid" theorem. $\dagger$

Theorem VI. If $a C_{n, r}^{\nu}$ and $a C_{n, r}^{\nu-\kappa}$ are chiastic there are $\infty^{\alpha l-r^{2}} C_{n, r}^{\nu-l} \mathrm{~s}$ $(\kappa>l)$ chiastic with both the $C_{n, r}^{\nu}$ and the $C_{n, r}^{\nu-\kappa}$. If we fix arbitrarily one $S_{r-l-1}$ of the $C_{n, r}^{\nu-l}$ lying in any $S_{r-1}$ of the $C_{n, r}^{\nu}$ and passing through the corresponding $S_{r-\kappa-1}$ of the $C_{n, r}^{\nu-\kappa}$, the $C_{n, r}^{\nu-l}$ is then completely determined.

TheORem VII. There are $\infty^{\kappa(\nu-\kappa+1)} C_{n, r}^{\nu-\kappa^{\prime}}$ s chiastic with any given $C_{n, r}^{\nu}$. We may fix arbitrarily the $\nu-\kappa+1$ points of the $C_{n, r}^{\nu-\kappa}$ lying on the

\footnotetext{
* The word chiastic is similarly used by Sir Robert Ball.

† Loc. cit., p. 171 ; also Grundzüge der Geometrie, p. 614.
} 
$\nu-\kappa+1 S_{\kappa}^{\prime}$ 's passing through any $S_{\kappa-1}$ of the $C_{n, r}^{\nu}$, and the $C_{n, r}^{\nu-\kappa}$ is then completely determined.

TheOREM VIII. There are $\infty^{\kappa(n-\nu+r-\kappa)} C_{n, r}^{\nu+\kappa} s$ chiastic with any given $C_{n, r}^{\nu}$. We may fix arbitrarily tike $n-\nu+r-\kappa$ copoints of the $C_{n, r}^{\nu+\kappa}$ passing through the $n-\nu+r-\kappa S_{r-\kappa-1}$ 's lying in any $S_{r-\kappa}$ of the $C_{n, r}^{\nu}$, and the $C_{n, r}^{\nu+\kappa}$ will then be completely determined.

Theorem IX. A $C_{n, r}^{v}$ is determined by

$$
r(n-\nu+r)+(\nu-r)(n-\nu+r-1)=n \nu-(\nu+1)(\nu-r)
$$

arbitrary constants.

\section{§ 2. Constructions of Quadric Polar Systems.}

It is well known that the Desargues configuration in the plane, the $\Gamma_{5,2}^{3}$, determines a certain conic or polar system with respect to which it is self-polar. Veronese shows, ${ }^{*}$ more generally, that the complete figure of two perspective "fundamental pyramids" in $S_{r}$, i. e., the configuration $\Gamma_{r+3, r}^{r+1}$, determines a certain $(r-1)$ dimensional quadric spread, $\Phi_{r-1}$, with respect to which it is selfpolar. Each point of the $\Gamma_{r+3, r}^{r+1}$, named by two letters is the pole, with respect to $\Phi_{r-1}$, of that particular copoint which is named by the remaining $r+1$ letters.

Let us consider first the case of the plane figure. CaYley shows $\dagger$ that the $\Gamma_{5,2}^{3}$ breaks up into two pentagons, each inscribed and circumscribed to the other. If we take any cyclic arrangement of the five letters, as $a b c d e(=b c d e a=e d c b a$, etc.), it will represent one of these pentagons, i. e., the pentagon whose vertices are $a b, b c, c d, d e$, and $e a$, and whose sides are $a b c, b c d, c d e$, dea, and $e a b$. The remaining five points and five lines of the configuration form the other pentagon, i. e., the pentagon acebd. In either of these pentagons, each vertex is the pole of the opposite side with respect to the polarity, $\Phi_{1}$, of the configuration.

To say that a quadric polarity sends each vertex of a pentagon into the opposite side places five linear conditions on the polarity, which is just sufficient to determine it uniquely. $\neq A$ polarity may then be given by a pentagon.

Of the two pentagons which form a $\Gamma_{5,2}^{3}$, one may be taken arbitrarily, and any line or point of the second to satisfy a single condition. (A $\Gamma_{5,2}^{3}$ depends

* Loc. cit., p. 193.

†Crelle's Journal, vol. 31 (1846), p. 213 ; also Collected Works, vol. I, p. 317. This fact was probably fist noticed by J. T. Graves. Cf. Philosophical Magazine, vol. 15 (1839), p. 131.

$\ddagger$ Cf. REYE, Geometrie der Lage (3d edition), rol. 2, p. 125 ; and KoHN, Mathematische Annalen, vol. 46 (1895), p. 303. 
upon $3.5-(3+1)(3-2)=11$ arbitrary constants. * Ten of these constants are fixed in fixing the first pentagon, leaving one arbitrary constant to the second pentagon). But the polarity is determined as soon as the first pentagon is fixed, hence the second pentagon is necessarily self-polar with respect to the polarity determined by the first pentagon.

This gives a method for constructing the polar of a given point with respect to a polarity, when the polarity is given by a self polar pentagon.

Consider the given pentagon as the pentagon $a b c d e$ of a $\Gamma_{5,2}^{3}$. If we make any side, say $a c e$, of the second pentagon, acebd, pass through the given point, $x$, this second pentagon is then determined, and the polar of $x$ must pass through the point $b d$. Now draw a different second pentagon, $a^{\prime} c^{\prime} e^{\prime} b^{\prime} d^{\prime}$, making a different side, say $b^{\prime} d^{\prime} a^{\prime}$, pass through $x$. Then the polar of $x$ must pass through the point $c^{\prime} e^{\prime}$ of this pentagon, and is thus determined.

The essential part of the construction may be remembered conveniently as follows: Number the vertices and sides of the pentagon consecutively $1,2,3, \ldots, 10$, assigning to the vertices the odd, and to the sides the even numbers. Then join $x$ to 1 to meet 4 at $A, A$ to 7 to meet 10 at $B, B$ to 3 to meet 6 at $C$, and $C$ is then on the polar of $x$. Shift the numbers, replacing 3 by 1,4 by 2,5 by $3, \ldots, 2$ by 10 , and repeat the above process, obtaining thus a point $C^{\prime}$. Then $C C^{\prime}$ is the polar of $x$.

A dual construction gives the pole of a given line.

Two pentagons, representing two polarities $\Phi$ and $\Phi^{\prime}$, of which the first sends points into lines and the second sends lines into points, give a collineation. The common polar triangle of $\Phi$ and $\Phi^{\prime}$ is the fixed triangle of the collineation. If $A B C D E$ is a pentagon giving the polarity $\Phi$, the triangle $B, D,(\overline{A B} \cdot \overline{D E})$ is evidently a polar triangle of $\Phi . \dagger$ If then a given triangle, with vertices $a, b$, and $c$, and sides $\alpha, \beta$, and $\gamma$, is to be the fixed triangle of a collineation which is in addition to send a given point, $P$, into a given point, $P^{\prime}$, we can at once construct two pentagons which will give this collineation. Let $\delta$ be any line. Our two pentagons may be $P, a,(\beta \cdot \delta),(\delta \cdot \alpha), b$ and $P^{\prime}, a$, $(\beta \cdot \delta),(\delta \cdot \alpha), b$.

The two pentagons $A B C D E$ and $A B C D E^{\prime}$, where $E^{\prime}$ lies on $D E$, give a collineation in which the point $D$ and all points on the line $A B$ are fixed points, while $\overline{A B}$, and all lines through the point $D$, are fixed lines. This collineation sends $E$ into $E^{\prime}$, and it sends any point, $P$, into a point $P^{\prime}$ on the line $P D$, such that the cross ratio $P, P^{\prime}, D, \overline{A B}$ is equal to the cross ratio $E, E^{\prime}, D, \overline{A B}$.

ClebsCH $\ddagger$ calls attention to a collineation determined by a pentagon. With

* Cf. $₹ 1$, theorem IX.

† Cf. Reye, Geometrie der Lage (3d edition), vol. 2, p. 125.

$¥$ Ueher die Anwendung der quadratischen Substitution auf die Gleichungen 5ten Grades und die geometrische Theorie des ebenen Fünseits, Mathematische Annalen, vol. 4 (1871). 
the pentagon $A B C D E$ is naturally associated the pentagon of the diagonals, $A C E B D$, and these two pentagons give the CLEBSCH collineation.

Passing now to the three-dimensional case,* we have the configuration, $\Gamma_{6,3}^{4}$, which consists of two perspective tetrahedra with their center and plane of perspection. The points, lines and planes are denoted respectively by combinations of two, three and four letters out of six. The point $a b$ is the pole of the plane $c d e f$, and the lines $a b c$ and $d e f$ are conjugate, with respect to a certain polarity $\Phi_{2}$.

Taking any cyclic arrangement of the six letters, as abcdef, they represent a skew-hexagon whose six vertices are $a b, b c, c d, d e$, ef and $f a$; six lines are $a b c, b c d, c d e, d e f, e f a$ and $f a b$; and six planes are $a b c d, b c d e, c d e f, d e f a$, $e f a b$ and $f a b c$.

In addition to the six vertices of this hexagon there are nine other points of the configuration, viz.,

$$
a c, c e, e a, b d, d f, f b, a d, b e \text { and } c f,
$$

each of which lies in two planes of the hexagon, the first six lying on lines and the last three on the intersection of opposite planes of the hexagon. (For instance, $a c$ lies on the line $a b c$, but $a d$ lies on the intersection of $a b c d$ and $d e f a$.) Similarly there are nine planes of the configuration besides the six of the hexagon, viz.,

$$
a b c e, b c d f, c d e a, d e f b, e f a c, f a b d, b c e f, c d f a \text { and } d e a b,
$$

the first six passing through lines of the hexagon, and the last three through the joins of opposite vertices.

From these nine points and nine planes we can pick out, in three different ways, a second hexagon which, like the first, may be represented by a cyclic arrangement of the six letters. Such a hexagon is adfbec, and, like the first hexagon, each vertex is the pole of the opposite plane with respect to the polarity $\Phi_{2}$. Moreover the hexagons $a b c d e f$ and $a d f b e c$ are mutually related to each other. Of the six vertices of each, four lie on lines, and two on the intersection of opposite planes of the other. Of the six planes of each, four pass through the lines, and two through the joins of opposite vertices of the other. Also four out of the six lines of each lie in planes and pass through vertices of the other.

To say that each vertex of a hexagon is the pole of the opposite plane with respect to a polarity $\Phi_{2}$ is to place nine linear conditions upon the polarity, and this determines it uniquely. Hence, as soon as one of the hexagons of our configuration is given, the polarity $\Phi_{2}$ is determined. $\dagger$

\footnotetext{
* Cf. the author's paper in The Johns Hopkins University Circular, April, 1904.

† Dr. KASNER, in a paper on The double-six configuration, A merican Journal, vol. 25 (1903), calls attention to a polarity, $\Omega$, connected with the double-six. In a letter to Professor Morley, he shows that a skew-hexagon, self-conjugate as to $\Omega$ (and thus determining $\Omega$ ) may be
} 
I wish now to show that the first hexagon, abcdef, can be taken arbitrarily, and any plane of the second hexagon, adfbec, can be taken to pass through any point in space, and that the second hexagon and the entire $\Gamma_{6,3}^{4}$ will then be determined.

Suppose abcdef to have been chosen arbitrarily. We then make any plane, say $a d f b$, of the second hexagon pass through the line $f a b$ and any given point $x$. Let $a d f b$ cut $d e f$ in $d f$ and $b c d$ in $b d$. Then draw

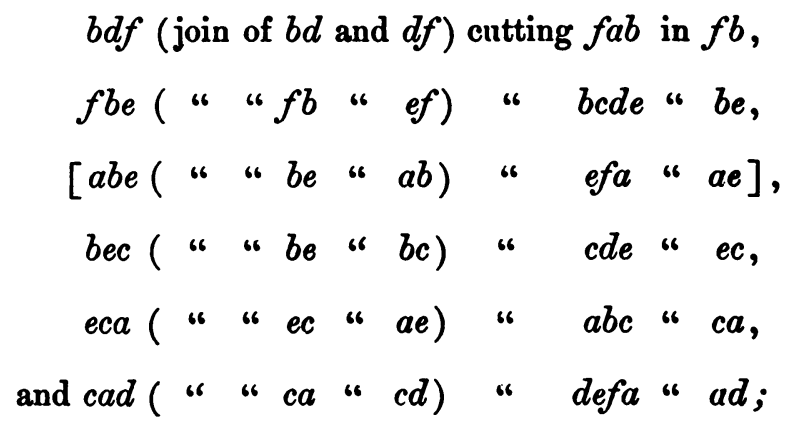

and if our hexagon is now to close properly, ad should lie in the plane $a d f b$ on a line with $f a$ and $d f$; and I shall show that this will always be the case.

The figure thus far constructed is part of the figure of two perspective tetrahedra, which we proceed to complete.

(In Fig. 1 the hexagon abcdef is shown by dotted lines, the hexagon adfbec by heavy lines, and the rest of the configuration by light lines. The double figure viewed through a stereoscope shows the figure in relief.)

Draw cef (join of $e c$ and $e f$ ). Then the three lines $f b c(f b \cdot b c), f a c$ $(f a \cdot c a)$, and $c d f(c d \cdot d f)$ will cut cef; for they lie respectively in the three planes $f b c e(f b \cdot b e \cdot e f \cdot b c \cdot e c)$, face $(f a \cdot a e \cdot e f \cdot c a \cdot e c)$, and $f c d e$ $(d f \cdot d e \cdot e f \cdot c d \cdot e c)$ passing through $c e f$. Moreover these three lines all cut $c e f$ at the same point, $c f$, because each pair of them lies in a plane which does not contain $c e f$; viz., $f b c$ and $f a c$ lie in $f a b c(f a \cdot f b \cdot a b \cdot b c \cdot c a)$; fac and $c d f$ lie in $f a c d(f a \cdot d f \cdot a d \cdot c d \cdot a c)$; and $c d f$ and $f b c$ lie in $f b c d(f b \cdot d f \cdot b d \cdot c d \cdot b c)$. Also the line $b d e$ ( $(b e \cdot d e)$ passes through $b d$ since it lies in two planes containing $b d$, viz., the planes $b d e f(b e \cdot d e \cdot f b \cdot d f \cdot b d)$ and $b c d e(b e \cdot d e \cdot b c \cdot d c \cdot b d)$.

Examination of the figure now shows that $c a b c c d c f$ and ae be de ef are two perspective tetrahedra with $e c$ as perspection-center. Five pairs of corresponding edges meet in the five points $b d, f b, d f, f a$, and $a b$, points in the plane

selected in twenty different ways from the lines of the double-six. If $L_{r}$ and $M_{i}(i=1,2, \ldots, 6)$ are the lines of the double-six, then any corresponding triples, as $L_{1}, L_{2}, L_{3}$ and $M_{1}, M_{2}, M_{2}$ form such a hexagon. In this same letter Dr. KAsNrr suggest a construction for a polarity when defined by a hexagon. He reduces it to the conetruotion of several plane polarities defined by plane pentagons. 
$a d f b$. The sixth pair of edges, cad and ade (ae $\cdot d e)$ must also meet in a point of this plane. But cad was to meet the plane defa in $a d$, and the line ade is

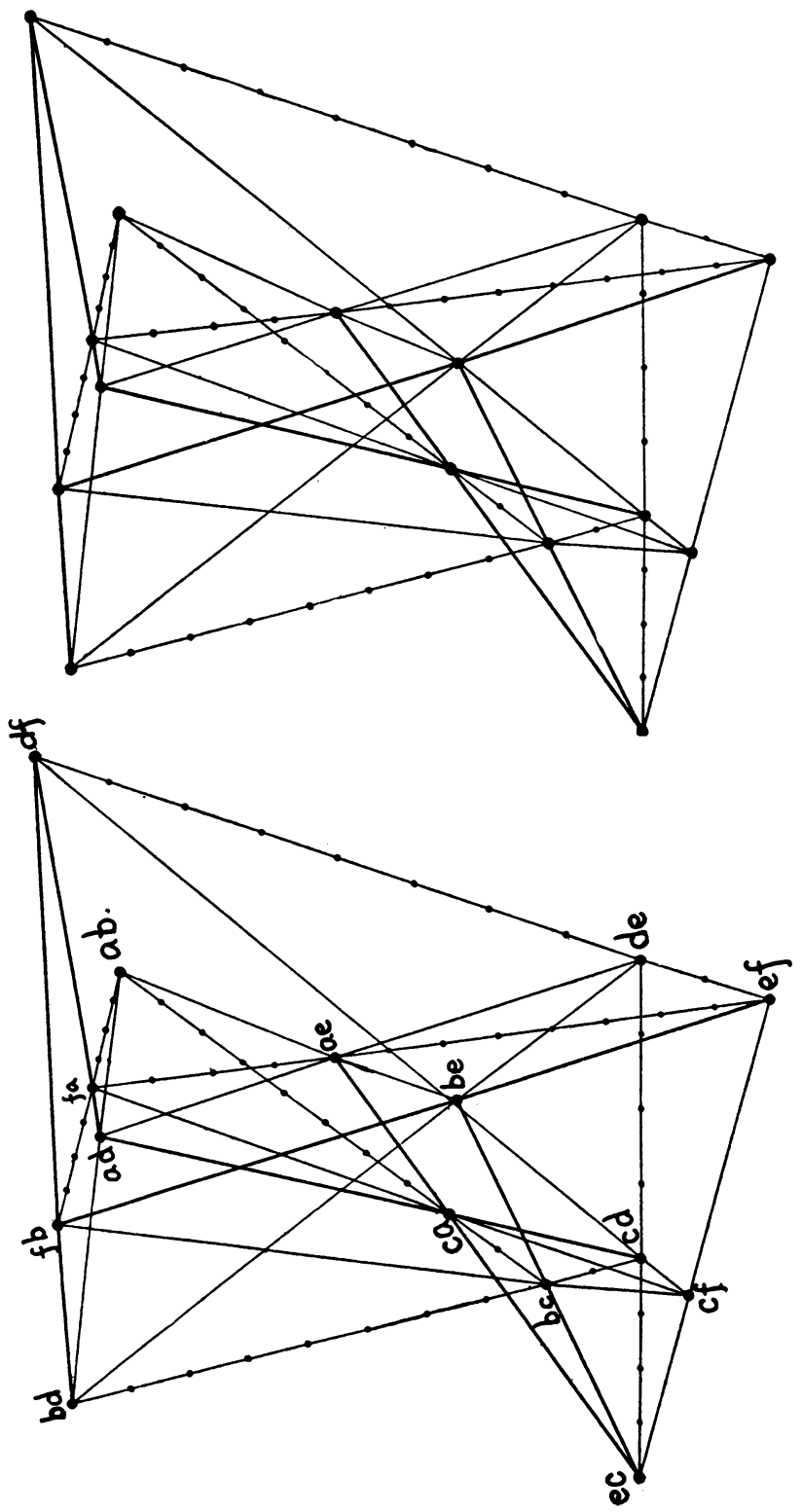

Fig. 1.

in the plane $d e f a$, and, therefore, $a d$ lies in the plane $a d f b$ and on a line with $f a$ and $d f$. Hence our hexagon closes properly, and the entire $\Gamma_{6,3}^{4}$ is determined. 
This gives us now a method, similar to the method for the plane, of constructing the polar plane of any point $x$ with respect to a polarity when the latter is given by a self-polar hexagon. The vertex of the second hexagon opposite to the plane passed through $x$ (the vertex $e c$ in the case just described) must lie on the polar plane of $x$. The construction of this point $e c$ is in fact very simple, and may be stated as follows :

Let $1,2,3,4,5,6$ be the vertices of the given hexagon taken in order. Pass a plane through $x, 1$, and 2 and cutting $\overline{34}$ and $\overline{56}$ in $A$ and $A^{\prime}$ respectively. Draw $\overline{A A^{\prime}}$ cutting $\overline{12}$ in $B$, and $B 6$ cutting the plane $\overline{345}$ in $C$. $\overline{C 3}$ will then cut $\overline{45}$ in the point $D$ which is the point required on the polar plane of $x$. Now shift the digits around on the hexagon, replacing 2 by 1,3 by $2, \ldots 1$ by 6 , and repeat the above process, obtaining a second point $D^{\prime}$, similarly for a third point $D^{\prime \prime}$. The plane $D D^{\prime} D^{\prime \prime}$ will then be the polar of $x$ with respect to the polarity given by the hexagon.

A dual construction gives the polar point of a given plane.

The combination of two consecutive space polarities gives a space collineation, and hence a space collineation may be given by two skew-hexagons.

If a given tetrahedron with vertices $a, b, c$, and $d$, and faces $\alpha, \beta, \gamma$, and $\delta$ is to be the fixed tetrahedron of a collineation which is in addition to send a given point, $P$, into a given point, $P^{\prime}$, this collineation may be given by the two skew hexagons whose vertices are

and

$$
P, a,(\gamma \cdot \beta \cdot \epsilon),(\beta \cdot \alpha \cdot \epsilon),(\alpha \cdot \delta \cdot \epsilon), b
$$

$$
P^{\prime}, a,(\gamma \cdot \beta \cdot \epsilon),(\beta \cdot \alpha \cdot \epsilon),(\alpha \cdot \delta \cdot \epsilon), b
$$

where $\epsilon$ is any plane.

The two hexagons $A B C D E F$ and $A B C D E F^{\prime}$, where $F^{\prime}$ lies on $E F$, give a collineation in which the point $E$ and all points of the plane $\overline{A B C}$ are fixed points, while the plane $A B C$ and all planes through $E$ are fixed planes. This collineation sends any point, $P$, into a point, $P^{\prime}$, on the line $P E$, such that the cross-ratio $P, P^{\prime}, E, \overline{A B} \bar{C}$ is equal to the cross-ratio $F, F^{\prime} E, A B C$.

\section{§3. Associated systems of conics.}

The two dual configurations, $\Gamma_{6,2}^{4}$ and $C_{6,2}^{4}$ (which are respectively the section by a plane and the projection on a plane of the space configuration $\Gamma_{6,3}^{4}$ ) give rise to certain peculiar sets of conics which are worthy of notice.

All the elements of a $\Gamma_{6,2}^{4}$ containing a given letter of the six, say $a$, form a $\Gamma_{5,2}^{3}{ }^{*}$ with which is associated a conic that may be called $F_{a}$. There are six such conics. The twenty points of the $\Gamma_{6,2}^{+}$naturally fall into pairs such as $a b c$

\footnotetext{
${ }^{*}$ Cf. \& 1, theorem I.
} 
and def. The point $a b c$ is the pole of the line adef with respect to $F_{a}$, and since def lies on $a d e f, a b c$ and def are a pair of conjugate points with respect to $F_{a}$. Similarly, since $a b c$ and $b d e f$ are pole and polar with respect to $F_{b}$, $a b c$ and def are conjugate with respect to $F_{b}$. They are conjugate points, in fact, with respect to all six $F$ 's. The same is true of each of the ten pairs of points. Regarding these ten point-pairs as degenerate line conics, and the $F$ 's as point conics, we have then ten line conics each apolar with each of six pointconics.

This being the case, either (1) the ten conics belong to a range and the six to a 4-spread; or (2) the ten belong to a web and the six to a net; or (3) the ten belong to a 4-spread and the six to a pencil. The first supposition cannot be true, for there can be only three degenerate conics in a range. If the second alternative be true, the twenty points lie on a cubic curve; but this is impossible since they lie by fours on straight lines. Therefore the third supposition is true, and the six conics $F$ belong to a pencil, i. e., they pass through four points. Thus every $\Gamma_{6,2}^{4}$ determines four covariant points.

In the same way it may be shown that a $C_{6,2}^{4}$ determines six line conics, $\Phi_{a}, \cdots, \Phi_{f}$, belonging to a range ; and hence $a C_{6,2}^{4}$ determines four covariant lines.

Every $\Gamma_{n, 2}^{\nu}$ (where $\nu \geqq 3$ and $n \geqq \nu+2$ ) contains $\left(\begin{array}{c}n \\ n-5\end{array}\right)\left(\begin{array}{c}n-5 \\ \nu-3\end{array}\right) \Gamma_{5,3}^{8}$ 's, with each of which is associated a conic $F$.* $^{*}$ We obtain one of these $\Gamma_{5,2}^{3}$ 's by picking out all the elements of the $\Gamma_{n, 2}^{\nu}$ which contain certain $\nu-3$ letters, say $a b \cdots \kappa$, and do not contain certain $n-\nu-2$ letters, say $m n \cdots s$. We may denote the conic associated with this $\Gamma_{5,2}^{3}$ by $F_{a b \ldots k m n} \ldots .$. If $\nu \geqq 4$, the configuration $\Gamma_{n, 2}^{\nu}$ contains $\left(\begin{array}{c}n \\ n-6\end{array}\right)\left(\begin{array}{c}n-6 \\ \nu-4\end{array}\right) \Gamma_{6,2}^{+}$'s, with each of which is associated a pencil of six of the conics $F$. The $\Gamma_{6,2}^{4}$, and therefore the associated pencil, may be denoted by the symbol $(b \cdots \kappa \cdot m n \cdots s)$ made up of two groups of $\nu-4$ and $n-\nu-2$ letters respectively. A given conic, as $F_{a b \ldots \kappa \cdot m n} \ldots$. belongs to a given pencil, as $(b \cdots \kappa \cdot m n \cdots s)$, if the $n-\nu+2$ letters in the two symbols are the same, and if the $\nu-4$ letters in the symbol for the pencil are contained among the $\nu-3$ letters in the symbol for the conic. We have then, connected with a $\Gamma_{n, 2}^{\nu}$ (where $\nu \geqq 4$ and $\left.n \geqq \nu+2\right),\left(\begin{array}{c}n \\ n-5\end{array}\right)\left(\begin{array}{c}n-5 \\ \nu-3\end{array}\right)$ conics lying in $\left(\begin{array}{c}n \\ n-6\end{array}\right)\left(\begin{array}{c}n-6 \\ \nu-4\end{array}\right)$ pencils, six conics in each pencil, and each conic in $\nu-3$ pencils.

Similarly, a $C_{n, 2}^{\mu}$ (where $\mu \geqq 4$ and $n \geqq \mu+2$ ) gives rise to $\left(\begin{array}{c}n \\ n-5\end{array}\right)\left(\begin{array}{c}n-5 \\ \mu-3\end{array}\right)$ lineconics lying in $\left(\begin{array}{c}n \\ n-6\end{array}\right)\left(\begin{array}{c}n-6 \\ \mu-4\end{array}\right)$ ranges, six conics in each range and each conic in $\mu-3$ ranges.

Restating this last theorem in terms of the $\Gamma$ 's instead of the $C$ 's, and noting that $\mu=n-\nu+1$, we have :

Associated with every $\Gamma_{n, 2}^{\nu}$ (where $\nu \geqq 3$ and $n \geqq \nu+3$ ) are $\left(\begin{array}{c}n \\ n-5\end{array}\right)\left(\begin{array}{c}n-5 \\ \nu-3\end{array}\right)$ lineconics lying in $\left(\begin{array}{c}n \\ n-8\end{array}\right)\left(\begin{array}{c}n-6 \\ \nu-3\end{array}\right)$ ranges, six conics in each range, and each conic in

\footnotetext{
* Ce. $\$ 1$, theorem IV.
} 
$n-\nu-2$ ranges. But these $\left(\begin{array}{c}n \\ n-5\end{array}\right)\left(\begin{array}{l}n-5 \\ \nu-3\end{array}\right)$ line-conics are the same as the point conics $F$ associated with the $\Gamma_{n, 2}^{v}$. Hence we have the somewhat remarkable theorem :

Associated with every $\Gamma_{n, 2}^{\nu}($ wnere $\nu \geqq 4$ and $n \geqq \nu+3)$ gre $\left(\begin{array}{c}n \\ n-5\end{array}\right)\left(\begin{array}{c}n-5 \\ \nu-3\end{array}\right)$ conics which lie by sixes in $\left(\begin{array}{c}n \\ n-6\end{array}\right)\left(\begin{array}{c}n-6 \\ \nu-4\end{array}\right)$ pencils, each conic in $\nu-3$ pencils, and which also lie by sixes in $\left(\begin{array}{c}n \\ n-6\end{array}\right)\left(\begin{array}{c}n-6 \\ \nu-3\end{array}\right)$ ranges, each soric in $n-\nu-2$ ranges. Colleg eville, Pa. 